\title{
ALGEBRAIC PERIODS OF SELF-MAPS OF A RATIONAL EXTERIOR SPACE OF RANK 2
}

\author{
GRZEGORZ GRAFF
}

Received 29 November 2004; Revised 27 January 2005; Accepted 21 July 2005

The paper presents a complete description of the set of algebraic periods for self-maps of a rational exterior space which has rank 2 .

Copyright (c) 2006 Grzegorz Graff. This is an open access article distributed under the Creative Commons Attribution License, which permits unrestricted use, distribution, and reproduction in any medium, provided the original work is properly cited.

\section{Introduction}

A natural number $m$ is called a minimal period of a map $f$ if $f^{m}$ has a fixed point which is not fixed by any earlier iterates. One important device for studying minimal periods are the integers $i_{m}(f)=\sum_{k / m} \mu(m / k) L\left(f^{k}\right)$, where $L\left(f^{k}\right)$ denotes the Lefschetz number of $f^{k}$ and $\mu$ is the classical Möbius function. If $i_{m}(f) \neq 0$, then we say that $m$ is an algebraic period of $f$. In many cases the fact that $m$ is an algebraic period provides information about the existence of minimal periods that are less then or equal to $m$. For example, let us consider $f$, a self-map of a compact manifold. If $f$ is a transversal map and odd $m$ is an algebraic period, then $m$ is a minimal period (cf. $[10,12]$ ). If $f$ is a nonconstant holomorphic map, then there exists $M>0$ such that for each prime number $m>M, m$ is a minimal period of $f$ if and only if $m$ is an algebraic period of $f$ (cf. [3]). Further relations between algebraic and minimal periods may be found in [8].

Sometimes the structure of the set of algebraic periods is a property of the space and may be deduced from the form of its homology groups. In [11] there is a description of algebraic periods for self-maps of a space $M$ with three nonzero (reduced) homology groups, each of which is equal to $\mathbb{Q}$, in [6] the authors consider a space $M$ with nonzero homology groups $H_{0}(M ; \mathbb{Q})=\mathbb{Q}, H_{1}(M ; \mathbb{Q})=\mathbb{Q} \oplus \mathbb{Q}$. The main difficulty in giving the overall description in the latter case is that for a map $f_{*}$ induced by $f$ on homology, for each $m$ there are complex eigenvalues for which $m$ is not an algebraic period. Rational exterior spaces are a wide class of spaces (e.g., Lie groups) which do not have this disadvantage, namely under the natural assumption of essentiality of $f$ there is a constant $m_{X}$ and computable set $T_{M}$, such that if $m>m_{X}, m \notin T_{M}$, then $m$ is an algebraic period of $f$ (cf. [5]). The aim of this paper is to provide a full characterization of algebraic periods 
in the case when homology spaces of $X$ are small dimensional, namely when $X$ is of the rank 2. Our work is based on $[1,9]$, where the description of the so-called "homotopical minimal periods" of self-maps of, respectively the two-, and three-dimensional torus are given using Nielsen numbers. We follow the algebraical framework of [9], the final description is similar to the one obtained in [1]. The differences result from the fact that the coefficients $i_{m}(f)$ are a sum of Lefschetz numbers, which unlike Nielsen numbers, do not have to be positive.

\section{Rational exterior spaces}

For a given space $X$ and an integer $r \geq 0$ let $H^{r}(X ; \mathbb{Q})$ be the $r$ th singular cohomology space with rational coefficients. Let $H^{*}(X ; \mathbb{Q})=\bigoplus_{r=0}^{s} H^{r}(X ; \mathbb{Q})$ be the cohomology algebra with multiplication given by the cup product. An element $x \in H^{r}(X ; \mathbb{Q})$ is decomposable if there are pairs $\left(x_{i}, y_{i}\right) \in H^{p_{i}}(X ; \mathbb{Q}) \times H^{q_{i}}(X ; \mathbb{Q})$ with $p_{i}, q_{i}>0, p_{i}+q_{i}=r>0$ so that $x=\sum x_{i} \cup y_{i}$. Let $A^{r}(X)=H^{r}(X) / D^{r}(X)$, where $D^{r}$ is the linear subspace of all decomposable elements.

Definition 2.1. By $A(f)$ we denote the induced homomorphism on $A(X)=\bigoplus_{r=0}^{s} A^{r}(X)$. Zeros of the characteristic polynomial of $A(f)$ on $A(X)$ will be called quotient eigenvalues of $f$. By $\operatorname{rank} X$ we will denote the dimension of $A(X)$ over $\mathbb{Q}$.

Definition 2.2. A connected topological space $X$ is called a rational exterior space if there are some homogeneous elements $x_{i} \in H^{\text {odd }}(X ; \mathbb{Q}), i=1, \ldots, k$, such that the inclusions $x_{i} \hookrightarrow H^{*}(X ; \mathbb{Q})$ give rise to a ring isomorphism $\Lambda_{\mathbb{Q}}\left(x_{1}, \ldots, x_{k}\right)=H^{*}(X ; \mathbb{Q})$.

Finite $H$-spaces including all finite dimensional Lie groups and some real Stiefel manifolds are the most common examples of rational exterior spaces. The two dimensional torus $T^{2}$, a product of two $n$-dimensional sphere $S^{n} \times S^{n}$, and the unitary group $U(2)$ are examples of rational exterior spaces of rank 2.

The Lefschetz number of self-maps of a rational exterior space can be expressed in terms of quotient eigenvalues.

Theorem 2.3 (cf. [7]). Let $f$ be a self-map of a rational exterior space, and let $\lambda_{1}, \ldots, \lambda_{k}$ be the quotient eigenvalues of $f$. Let $A$ denote the matrix of $A(f)$. Then $L\left(f^{m}\right)=\operatorname{det}\left(I-A^{m}\right)=$ $\prod_{i=1}^{k}\left(1-\lambda_{i}^{m}\right)$.

Remark 2.4. A basis of the space $A(X)$ may be chosen in such a way that the matrix $A$ is integral (cf. [7]).

\section{The set of algebraic periods of self-maps of rational exterior space of rank 2}

Let $\mu$ denote the Möbius function, that is, the arithmetical function defined by the three following properties: $\mu(1)=1, \mu(k)=(-1)^{r}$ if $k$ is a product of $r$ different primes, and $\mu(k)=0$ otherwise. Let $\operatorname{APer}(f)=\left\{m \in \mathbb{N}: i_{m}(f) \neq 0\right\}$, where $i_{m}(f)=\sum_{k / m} \mu(m / k) L\left(f^{k}\right)$. We will study the form of APer $(f)$ for $f: X \rightarrow X$ and $X$ a rational exterior space of rank 2 . We assume that $X$ is not simple which means that there exists $r \geq 1$ such that $\operatorname{dim} A^{r}=2$, otherwise, that is, if there are $i, j \geq 1$ such that $\operatorname{dim} A^{i}=\operatorname{dim} A^{j}=1$, we get the case with 
Table 3.1. The set of algebraic periods $\operatorname{APer}(f)$ for the set $R$.

\begin{tabular}{l|cc}
\hline No. & $(t, d)$ & $\operatorname{APer}(f)$ \\
\hline $1^{0}$ & $(-2,1)$ & $\{1,2\}$ \\
$2^{0}$ & $(-1,0)$ & $\{1,2\}$ \\
$3^{0}$ & $(0,0)$ & $\{1\}$ \\
$4^{0}$ & $(0,1)$ & $\{1,2,4\}$ \\
$5^{0}$ & $(1,1)$ & $\{1,2,3,6\}$ \\
$6^{0}$ & $(-1,1)$ & $\{1,3\}$ \\
\hline
\end{tabular}

integer quotient eigenvalues (cf. [7]) for which the description of $\operatorname{APer}(f)$ easily follows from the case under consideration.

By Theorem 2.3 we see that $A$ is a $2 \times 2$ matrix and that the Lefschetz numbers $L\left(f^{m}\right)$ are expressed by its two quotient eigenvalues (in short we will call them eigenvalues): $\lambda_{1}, \lambda_{2}: L\left(f^{m}\right)=\left(1-\lambda_{1}^{m}\right)\left(1-\lambda_{2}^{m}\right)$. The characteristic polynomial of $A$ has integer coefficients by Remark 2.4 and is given by the formula: $W_{A}(x)=x^{2}-t x+d$, where $t=$ $\lambda_{1}+\lambda_{2}$ is the trace of $A$ and $d=\lambda_{1} \lambda_{2}$ is its determinant. The characterization of the set $\operatorname{APer}(f)$ will be given in terms of these two parameters: $t$ and $d$. Let us define the set $R=\{(-2,1),(-1,0),(0,0),(0,1),(1,1),(-1,1)\}$.

THeOREM 3.1. Let $f$ be a self-map of a rational exterior space $X$ of rank 2, which is not simple. Then $\operatorname{APer}(f)$ is one of the three mutually exclusive types:

(E) $\operatorname{APer}(f)$ is empty if and only if 1 is an eigenvalue of $A$, which is equivalent to $t-d=1$.

(F) $\operatorname{APer}(f)$ is nonempty but finite if and only if all the eigenvalues of $A$ are either zero or roots of unity not equal to 1 , which is equivalent to $(t, d) \in R$. The algebraic periods for the set $R$ are given in Table 3.1. then,

(G) $\operatorname{APer}(f)$ is infinite. Assume that $(t, d)$ is not covered by the types $(E)$ and $(F)$,

(1) for $(t, d)=(-2,2), \operatorname{APer}(f)=\mathbb{N} \backslash\{2,3\}$;

(2) for $(t, d)=(-1,2), \operatorname{APer}(f)=\mathbb{N} \backslash\{3\}$;

(3) for $(t, d)=(0,2), \operatorname{APer}(f)=\mathbb{N} \backslash\{4\}$;

(4) for $t=-d$ and $(t, d) \neq(-2,2), \operatorname{APer}(f)=\mathbb{N} \backslash\{2\}$;

(5) for $t+d=-1, \operatorname{APer}(f)=\mathbb{N} \backslash\{n \in \mathbb{N}: n \equiv 0(\bmod 4)\}$;

(6) if $(t, d)$ is not covered by any of the cases $1-5$, then $\operatorname{APer}(f)=\mathbb{N}$.

Remark 3.2. The letters E, F, G are chosen to represent empty, finite and generic case, respectively, which corresponds to the notation used in [9].

The rest of the paper consists of the proof of Theorem 3.1 and is organized in the following way: in the first part we describe the conditions equivalent to the fact that $m \in$ $\{1,2,3\}$ is not an algebraic period. In the second part we analyze the situation when $m>3$ and none of eigenvalues is a root of unity. This is done by considering two cases: we will study the behaviour of $i_{m}(f)$ separately for real and complex eigenvalues. In the third stage we consider the case when $m>3$ and one of eigenvalues is a root of unity. 
4 Algebraic periods for maps of rational exterior spaces

\subsection{Algebraic periods in $\{1,2,3\}$}

(A) Conditions for $1 \notin \operatorname{APer}(f)$. We have: $i_{1}(f)=L(f)=\left(1-\lambda_{1}\right)\left(1-\lambda_{2}\right)=0$. This may happen if and only if one of the eigenvalues is equal to 1 , that is, $t-d=1$.

(B) Conditions for $2 \notin \operatorname{APer}(f)$. We have: $i_{2}(f)=L\left(f^{2}\right)-L(f)=0$, which is equivalent to: $\left(1-\lambda_{1}^{2}\right)\left(1-\lambda_{2}^{2}\right)-\left(1-\lambda_{1}\right)\left(1-\lambda_{2}\right)=0$. This gives: $\left(1-\lambda_{1}\right)\left(1-\lambda_{2}\right)\left[\left(1+\lambda_{1}\right)\right.$ $\left.\left(1+\lambda_{2}\right)-1\right]=0$, so again $t-d=1$ or:

$$
\lambda_{1} \lambda_{2}+\lambda_{1}+\lambda_{2}=0
$$

which gives $d+t=0$. The conditions for $2 \notin \operatorname{APer}(f)$ are: $t-d=1$ or $t=-d$.

(C) Conditions for $3 \notin \operatorname{APer}(f)$. We have: $i_{3}(f)=L\left(f^{3}\right)-L(f)=0$, which is equivalent to: $\left(1-\lambda_{1}^{3}\right)\left(1-\lambda_{2}^{3}\right)-\left(1-\lambda_{1}\right)\left(1-\lambda_{2}\right)=0$. We obtain the following equation: $\left(1-\lambda_{1}\right)$ $\left(1-\lambda_{2}\right)\left[\left(1+\lambda_{1}+\lambda_{1}^{2}\right)\left(1+\lambda_{2}+\lambda_{2}^{2}\right)-1\right]=0$. Again $t-d=1$ if one of the eigenvalues is equal to 1 , otherwise:

$$
\lambda_{1}+\lambda_{2}+\lambda_{1} \lambda_{2}+\lambda_{1}^{2}+\lambda_{2}^{2}+\lambda_{1} \lambda_{2}\left(\lambda_{1}+\lambda_{2}\right)+\left(\lambda_{1} \lambda_{2}\right)^{2}=0
$$

In parameters $t$ and $d$ this gives:

$$
t^{2}+t-d+d t+d^{2}=0
$$

The last equality may be written as:

$$
\left(d-\frac{1-t}{2}\right)^{2}+\frac{3}{4}(1+t)^{2}=1,
$$

which leads to the following alternatives.

If $t=0$, then $d \in\{0,1\}$, which corresponds to characteristic polynomials $x^{2}=0\left(\lambda_{1}=\right.$ $\left.\lambda_{2}=0\right)$ and $x^{2}+1=0\left(\lambda_{1,2}= \pm i\right)$.

If $t=-1$, then $d \in\{0,2\}$, which corresponds to characteristic polynomials $x^{2}+x=0$ $\left(\lambda_{1}=0, \lambda_{2}=-1\right)$ and $x^{2}+x+2=0\left(\lambda_{1,2}=-(1 / 2) \pm i(\sqrt{7} / 2)\right)$.

If $t=-2$, then $d \in\{1,2\}$, which corresponds to characteristic polynomials $x^{2}+2 x+$ $1=0\left(\lambda_{1,2}=-1\right)$ and $x^{2}+2 x+2=0\left(\lambda_{1,2}=-1 \pm i\right)$.

The conditions for $3 \notin \operatorname{APer}(f)$ are: $t-d=1$ or $(t, d) \in\{(0,0),(0,1),(-1,0),(-1,2)$, $(-2,1),(-2,2)\}$.

3.2. Algebraic periods in the set $m>3$ in the case when none of the two eigenvalues is a root of unity. Let for the rest of the paper $\left|\lambda_{1}\right|=\max \left\{\left|\lambda_{1}\right|,\left|\lambda_{2}\right|\right\}$. We will need the following lemma.

LemMA 3.3. If for some $m$ and each $n \mid m, n \neq m$ we have $\left|L\left(f^{m}\right)\right| /\left|L\left(f^{n}\right)\right|>2 \sqrt{m}-1$, then $m$ is an algebraic period. 
Proof. Let $\left|L\left(f^{s}\right)\right|=\max \left\{\left|L\left(f^{l}\right)\right|: l \mid m, l \neq m\right\}$. We have

$$
\begin{aligned}
\left|i_{m}(f)\right| & =\left|\sum_{l \mid m} \mu\left(\frac{m}{l}\right) L\left(f^{l}\right)\right| \geq\left|L\left(f^{m}\right)\right|-\left|\sum_{l \mid m, l \neq m} \mu\left(\frac{m}{l}\right) L\left(f^{l}\right)\right| \\
& \geq\left|L\left(f^{m}\right)\right|-(2 \sqrt{m}-1)\left|L\left(f^{s}\right)\right| .
\end{aligned}
$$

The last inequality is a consequence of the fact that the number of different divisors of $m$ is not greater than $2 \sqrt{m}$ (cf. [2]), by the assumption we get $\left|i_{m}(f)\right|>0$, which is the desired assertion.

Now, using the algebraic arguments of [9] in a case of two eigenvalues, we find the bound for the ratio $\left|L\left(f^{m}\right)\right| /\left|L\left(f^{n}\right)\right|$. We have

$$
\frac{\left|L\left(f^{m}\right)\right|}{\left|L\left(f^{n}\right)\right|}=\frac{\left|1-\lambda_{1}^{m}\right|\left|1-\lambda_{2}^{m}\right|}{\left|1-\lambda_{1}^{n}\right|\left|1-\lambda_{2}^{n}\right|} \geq \frac{\left|\lambda_{1}\right|^{m}-1}{\left|\lambda_{1}\right|^{n}+1} \frac{\left|\lambda_{2}\right|^{m}-1}{\left|\lambda_{2}\right|^{n}+1}
$$

Let us consider two cases.

Case 1. $\lambda_{1}, \lambda_{2}$ are complex conjugates, then $\left|\lambda_{1}\right|=\left|\lambda_{2}\right|$. Notice that $\left|\lambda_{1}\right|=\sqrt{d}$, so if we exclude three pairs $(t, d) \in\{(0,1),(-1,1),(1,1)\}$, which correspond to some roots of unity, we obtain: $\left|\lambda_{1}\right|>1.4$.

Let $n \mid m$, for Lefschetz numbers in this case we have

$$
\frac{\left|L\left(f^{m}\right)\right|}{\left|L\left(f^{n}\right)\right|} \geq\left(\left|\lambda_{1}\right|^{m / 2}-1\right)\left(\left|\lambda_{2}\right|^{m / 2}-1\right)=\left(\left|\lambda_{1}\right|^{m / 2}-1\right)^{2} .
$$

Case 2. $\lambda_{1}, \lambda_{2}$ are real. Then $\left|\lambda_{1}\right|=\left(|t|+\sqrt{t^{2}-4 d}\right) / 2$. If $(t, d)=(0,0)$ then we immediately have $\operatorname{APer}(f)=\{1\}$. Cases $t=0, d=-1$ and $t= \pm 1, d=0$ and $t= \pm 2, d=1$ give some roots of unity. In the rest of the cases: $\left|\lambda_{1}\right| \geq 1.4$.

In order to obtain the estimation for Lefschetz numbers we use the following inequality for the moduli of eigenvalues (cf. [9, Lemma 5.2]).

Lemma 3.4. Let $\lambda_{i} \neq \pm 1, i=1,2$, then

$$
|1-| \lambda_{2}|| \geq \frac{1}{1+\left|\lambda_{1}\right|} .
$$

Proof. $\left|\left( \pm 1-\lambda_{1}\right)\left( \pm 1-\lambda_{2}\right)\right|=\left|W_{A}( \pm 1)\right| \geq 1$, because both eigenvalues are different from \pm 1 . We obtain $\left|1 \pm \lambda_{2}\right| \geq 1 /\left|1 \pm \lambda_{1}\right| \geq 1 /\left(1+\left|\lambda_{1}\right|\right)$, which gives the needed inequality.

We have by Lemma 3.4: $\left|\lambda_{2}\right|-1 \geq\left(\left|\lambda_{1}\right|+1\right)^{-1}$ for $\left|\lambda_{2}\right|>1$ and $1-\left|\lambda_{2}\right| \geq\left(\left|\lambda_{1}\right|+1\right)^{-1}$ for $\left|\lambda_{2}\right|<1$.

Let $h(x)=\left(x^{m}-1\right) /\left(x^{n}+1\right)$, notice that $h(x)$ is an increasing and $-h(x)$ is a decreasing function for $m>n>0$ and $x>0$.

Taking into account the two facts mentioned above we obtain:

$$
\frac{\left|1-\lambda_{2}^{m}\right|}{\left|1-\lambda_{2}^{n}\right|} \geq \min \left\{\frac{\left[1+\left(\left|\lambda_{1}\right|+1\right)^{-1}\right]^{m}-1}{\left[1+\left(\left|\lambda_{1}\right|+1\right)^{-1}\right]^{n}+1}, \frac{1-\left[1-\left(\left|\lambda_{1}\right|+1\right)^{-1}\right]^{m}}{1+\left[1-\left(\left|\lambda_{1}\right|+1\right)^{-1}\right]^{n}}\right\} .
$$


As $n \mid m$ we get

$$
\frac{\left|L\left(f^{m}\right)\right|}{\left|L\left(f^{n}\right)\right|} \geq\left(\left|\lambda_{1}\right|^{m / 2}-1\right) \min \left\{\left[1+\left(\left|\lambda_{1}\right|+1\right)^{-1}\right]^{m / 2}-1,1-\left[1-\left(\left|\lambda_{1}\right|+1\right)^{-1}\right]^{m / 2}\right\} .
$$

Let $\bar{f}_{C}\left(\left|\lambda_{1}\right|, m\right), \bar{f}_{R}\left(\left|\lambda_{1}\right|, m\right)$ be the functions equal to the right-hand side of the formulas (3.7) and (3.10), respectively. We define functions $f_{C}\left(\left|\lambda_{1}\right|, m\right)=\bar{f}_{C}\left(\left|\lambda_{1}\right|, m\right)-(2 \sqrt{m}-$ 1) and $f_{R}\left(\left|\lambda_{1}\right|, m\right)=\bar{f}_{R}\left(\left|\lambda_{1}\right|, m\right)-(2 \sqrt{m}-1)$. Notice that the inequalities:

$$
\begin{aligned}
& f_{C}\left(\left|\lambda_{1}\right|, m\right)>0, \\
& f_{R}\left(\left|\lambda_{1}\right|, m\right)>0,
\end{aligned}
$$

imply that $\left|L\left(f^{m}\right)\right| /\left|L\left(f^{n}\right)\right|>2 \sqrt{m}-1$ for $n \mid m$.

It is not difficult to verify the following statement by calculation and estimation of appropriate partial derivatives.

Remark 3.5. $f_{C}(\cdot, m)$ and $f_{C}\left(\left|\lambda_{1}\right|, \cdot\right)$ are increasing functions for $\left|\lambda_{1}\right|>1.4, m \geq 4$.

$f_{R}(\cdot, m)$ and $f_{R}\left(\left|\lambda_{1}\right|, \cdot\right)$ are increasing functions for $\left|\lambda_{1}\right|>1.4, m \geq 6$ and for $\left|\lambda_{1}\right| \geq 3$, $m \geq 4$.

If one of the inequalities (3.11), (3.12) is satisfied for given values $\left|\lambda_{1}^{0}\right|$ and $m_{0}$, then, by Remark 3.5, it is valid for each $\left|\lambda_{1}\right|>\left|\lambda_{1}^{0}\right|$ and $m>m_{0}$ and by Lemma 3.3 all such $m>m_{0}$ are algebraic periods.

LEMMA 3.6. Let us assume that both eigenvalues are complex

(a) if $m \geq 7$, then $m$ is an algebraic period,

(b) if $\left|\lambda_{1}\right| \geq 2$ and $m \geq 4$, then $m$ is an algebraic period.

Proof. We take the minimal modulus of the eigenvalue which may appear and put it in the formula (3.11): (a) $f_{C}(1.4,7)>0.75$, (b) $f_{C}(2,4)=6$, which gives the result by Remark 3.5.

LEMmA 3.7. Let us assume that both eigenvalues are real

(a) if $m \geq 12$, then $m$ is an algebraic period,

(b) if $\left|\lambda_{1}\right| \geq 3$ and $m \geq 6$, then $m$ is an algebraic period.

Proof. We put in the formula (3.12) the minimal modulus of the greater eigenvalue: (a) $f_{R}(1.4,12)>0.59$, (b) $f_{R}(3,6)>17.47$, which implies the result by Remark 3.5.

Remark 3.8. We must only check the cases when $\left|\lambda_{1}\right|<3$ and $4 \leq m \leq 11$. Notice that for the coefficients $t, d$ of the characteristic polynomial $W_{A}(x)$ we have the following estimates: $|t| \leq 2\left|\lambda_{1}\right|,|d| \leq\left|\lambda_{1}\right|^{2}$. This gives the bound: $|t|<6,|d|<9$, thus there are at most $11 \times 17 \times 8=1496$ cases which should be checked. This is done by numerical computation. If we exclude $(t, d)=(0,0)$ and the pairs which give the eigenvalues being roots of unity, we find in the range under consideration that only for $(t, d)=(0,2), m=4$ is not an algebraic period. 
3.3. Algebraic periods in the set $m>3$ in the case when one of the two eigenvalues is a root of unity. If both eigenvalues are real, then one of them is equal \pm 1 . If they are complex conjugates, then $\lambda_{1} \lambda_{2}=\lambda_{1} \bar{\lambda}_{1}=1$, thus $d=1$. On the other hand $0 \leq\left|\lambda_{1}+\lambda_{2}\right| \leq$ $\left|\lambda_{1}\right|+\left|\lambda_{2}\right|=2$, thus $|t| \leq 2$. This gives three pairs of complex eigenvalues: $\pm i(t=0, d=1)$ and $(1 / 2) \pm i(\sqrt{3} / 2)(t=1, d=1)$ and $-(1 / 2) \pm i(\sqrt{3} / 2)(t=-1, d=1)$. Each of these five cases we consider separately.

(1) 1 is one of eigenvalues $(t-d=1)$. Then $L\left(f^{m}\right)=0$ for all $m$ and consequently $i_{m}(f)=$ 0 for all $m$. Thus $\operatorname{APer}(f)=\varnothing$.

(2) -1 is one of eigenvalues $(t+d=-1)$. We have to consider the subcases.

(2a) If $d=-1$, then $t=0$, so we are in case 1 .

(2b) If $d=0$, then $t=-1$, so $W_{A}(x)=x^{2}+x$ and the second eigenvalue is equal to 0. $L\left(f^{m}\right)=1-(-1)^{m}$, thus $L\left(f^{m}\right)=0$ for $m$ even and $L\left(f^{m}\right)=2$ for $m$ odd. We get: $i_{m}(f)=\sum_{k: 2|k| m} \mu(m / k) L\left(f^{k}\right)+\sum_{k: 2 \nmid k \mid m} \mu(m / k) L\left(f^{k}\right)=2 \sum_{k: 2 \nmid k \mid m} \mu(m / k)$. It is easy to find (see the calculation of $i_{m}(f)$ in $(2 \mathrm{~d})$ ) that $i_{1}(f)=2, i_{2}(f)=-2$, $i_{m}(f)=0$ for $m \geq 3$. As a consequence: $\operatorname{APer}(f)=\{1,2\}$.

(2c) If $d=1$, then $t=-2$, so $W_{A}(x)=x^{2}+2 x+1$ and the second eigenvalue is equal to -1 . $L\left(f^{m}\right)=\left(1-(-1)^{m}\right)^{2}$, thus $L\left(f^{m}\right)=0$ for $m$ even and $L\left(f^{m}\right)=4$ for $m$ odd. We check in the same way as above that $i_{1}(f)=4, i_{2}(f)=-4, i_{m}(f)=0$ for $m \geq 3$, so $\operatorname{APer}(f)=\{1,2\}$.

(2d) If $d \in \mathbb{Z} \backslash\{-1,0,1\}$, then for each $m:\left|L\left(f^{m}\right)\right|=\left|\left(1-(-1)^{m}\right)\right|\left|1-\lambda_{1}^{m}\right|$. Notice that in the case under consideration $\{1,2,3\} \subset \operatorname{APer}(f)$, which follows from Section 3.1 .

As $|d|=\left|\lambda_{1}\right|\left|\lambda_{2}\right|$ and -1 is one of eigenvalues we obtain for $k$ odd : $\left|L\left(f^{k}\right)\right| \geq 2\left(\left|\lambda_{1}^{k}\right|-\right.$ $1)=2\left(|d|^{k}-1\right),\left|L\left(f^{k}\right)\right| \leq 2\left(\left|\lambda_{1}^{k}\right|+1\right)=2\left(|d|^{k}+1\right)$. Thus, for $m$ odd, estimating in the same way as in Lemma 3.3, we get:

$$
\left|i_{m}(f)\right| \geq 2\left(|d|^{m}-1\right)-(2 \sqrt{m}-1) 2\left(|d|^{m / 3}+1\right) .
$$

The right-hand side of the above formula is greater then zero for $|d| \geq 2, m>3$, so all odd $m>3$ are algebraic periods.

If $m>3$ is even, then $m=2^{n} q$, where $q$ is odd. By the fact that $L\left(f^{r}\right)=0$ if $2 \mid r$, we get $L\left(f^{2^{i} q}\right)=0$, for $1 \leq i \leq n$, thus

$$
i_{m}(f)=\sum_{l \mid 2^{n} q} \mu\left(2^{n} \frac{q}{l}\right) L\left(f^{l}\right)=\sum_{l \mid q} \mu\left(2^{n} \frac{q}{l}\right) L\left(f^{l}\right) .
$$

As $\mu$ is multiplicative and $\mu\left(2^{n}\right)=-1$ for $n=1$ and $\mu\left(2^{n}\right)=0$ for $n>1$, we get

$$
i_{m}(f)= \begin{cases}-i_{q}(f) & \text { if } n=1 \\ 0 & \text { if } n>1\end{cases}
$$

This leads to the conclusion that even $m$ is an algebraic period if and only if $m=2 q$ where $q$ is odd. Finally in the case $(2 d)$ we obtain

$$
\operatorname{APer}(f)=\mathbb{N} \backslash\{n \in \mathbb{N}: n \equiv 0(\bmod 4)\} .
$$


Before we consider complex cases let us state the following fact (cf. [4]). Let $g_{*}$, generated by $g$ on homology, have as its only eigenvalues $\varepsilon_{1}, \ldots, \varepsilon_{\phi(d)}$ which are all the $d$ th primitive roots of unity $(\phi(d)$ denotes the Euler function). Then the Lefschetz numbers of iterations of $g$ are the sum of powers of these roots: $L\left(g^{m}\right)=\sum_{i=1}^{\phi(d)} \varepsilon_{i}^{m}$. We have the formula for $i_{m}(g)$ in such a case:

$$
i_{m}(g)= \begin{cases}0 & \text { if } m \chi d \\ \sum_{k \mid m} \mu\left(\frac{d}{k}\right) \mu\left(\frac{m}{k}\right) \frac{\phi(d)}{\phi(d / k)} & \text { if } m \mid d .\end{cases}
$$

Let now $\lambda_{1,2}$ be complex conjugates eigenvalues, then

$$
L\left(f^{m}\right)=1-\lambda_{1}^{m}-\lambda_{2}^{m}+\left(\lambda_{1} \lambda_{2}\right)^{m}=2-\left(\lambda_{1}^{m}+\lambda_{2}^{m}\right) .
$$

We may rewrite formula for $L\left(f^{m}\right)$ in the following way: $L\left(f^{m}\right)=2-L\left(g^{m}\right)$, where $g$ is described above. As $\sum_{k \mid m} \mu(m / k) 2=2$ for $m=1$ and 0 for $m>1$; we get

$$
i_{m}(f)= \begin{cases}2-i_{m}(g) & \text { if } m=1, \\ -i_{m}(g) & \text { if } m>1\end{cases}
$$

(3) $\lambda_{1,2}= \pm i(t=0, d=1)$ are all primitive roots of unity of degree 4 . Thus, applying formula (3.17) and (3.19), we get $i_{1}(f)=2, i_{2}(f)=2, i_{3}(f)=0, i_{4}(f)=-4$, and $i_{m}(f)=$ 0 for $m>4$. Summing it up: $\operatorname{APer}(f)=\{1,2,4\}$.

(4) $\lambda_{1,2}=-1 / 2 \pm i(\sqrt{3} / 2)(t=1, d=1)$ are all the primitive roots of unity of degree 6. Again by formulas (3.17) and (3.19) we calculate the values of $i_{m}(f)$ and get: $i_{1}(f)=1, i_{2}(f)=2, i_{3}(f)=3, i_{4}(f)=0, i_{5}(f)=0, i_{6}(f)=-6$ and $i_{m}(f)=0$ for $m>6$, so $\operatorname{APer}(f)=\{1,2,3,6\}$.

(5) $\lambda_{1,2}=(1 / 2) \pm i(\sqrt{3} / 2)(t=-1, d=1)$ are all the primitive roots of unity of degree 3. By (3.17) and (3.19) we have: $i_{1}(f)=3, i_{2}(f)=0, i_{3}(f)=-3, i_{m}(f)=0$ for $m>3$, so $\operatorname{APer}(f)=\{1,3\}$.

\section{Acknowledgment}

Research supported by KBN Grant no. 2 P03A 04522.

\section{References}

[1] L. Alsedà, S. Baldwin, J. Llibre, R. Swanson, and W. Szlenk, Minimal sets of periods for torus maps via Nielsen numbers, Pacific Journal of Mathematics 169 (1995), no. 1, 1-32.

[2] K. Chandrasekharan, Introduction to Analytic Number Theory, Die Grundlehren der mathematischen Wissenschaften, vol. 148, Springer, New York, 1968.

[3] N. Fagella and J. Llibre, Periodic points of holomorphic maps via Lefschetz numbers, Transactions of the American Mathematical Society 352 (2000), no. 10, 4711-4730.

[4] G. Graff, Existence of $\delta_{m}$-periodic points for smooth maps of compact manifold, Hokkaido Mathematical Journal 29 (2000), no. 1, 11-21.

[5] _ Minimal periods of maps of rational exterior spaces, Fundamenta Mathematicae 163 (2000), no. 2, 99-115. 
[6] A. Guillamon, X. Jarque, J. Llibre, J. Ortega, and J. Torregrosa, Periods for transversal maps via Lefschetz numbers for periodic points, Transactions of the American Mathematical Society 347 (1995), no. 12, 4779-4806.

[7] D. Haibao, The Lefschetz numbers of iterated maps, Topology and its Applications 67 (1995), no. $1,71-79$.

[8] J. Jezierski and M. Marzantowicz, Homotopy Methods in Topological Fixed and Periodic Point Theory, Springer, Dordrech, 2005.

[9] B. Jiang and J. Llibre, Minimal sets of periods for torus maps, Discrete and Continuous Dynamical Systems 4 (1998), no. 2, 301-320.

[10] J. Llibre, Lefschetz numbers for periodic points, Nielsen Theory and Dynamical Systems (South Hadley, Mass, 1992), Contemp. Math., vol. 152, American Mathematical Society, Rhode Island, 1993, pp. 215-227.

[11] J. Llibre, J. Paraños, and J. A. Rodríguez, Periods for transversal maps on compact manifolds with a given homology, Houston Journal of Mathematics 24 (1998), no. 3, 397-407.

[12] W. Marzantowicz and P. M. Przygodzki, Finding periodic points of a map by use of a $k$-adic expansion, Discrete and Continuous Dynamical Systems 5 (1999), no. 3, 495-514.

Grzegorz Graff: Department of Algebra, Faculty of Applied Physics and Mathematics, Gdansk University of Technology, ul G. Narutowicza 11/12, 80-952, Gdansk, Poland E-mail address: graff@mifgate.pg.gda.pl 